\title{
Patterning Photosensitive Layers for Optoelectronic Applications
}

\author{
Istvan Csarnovics $^{1)}$, Sandor Molnar ${ }^{1)}$, Julia Burunkova ${ }^{2)}$, Dmitrii Zhuk ${ }^{2)}$, Igor Denisyuk ${ }^{2)}$, \\ Attila Bonyar ${ }^{3)}$, Csaba Cserhati ${ }^{1)}$, Sandor Kokenyesi*1,2) \\ ${ }^{1)}$ University of Debrecen, Institute of Physics, Debrecen, Hungary \\ ${ }^{2)}$ University ITMO, St. Petersburg, Russia \\ 3) Budapest University of Technology and Economics, Budapest, Hungary \\ ${ }^{*}$ kiki@science.unideb.hu
}

\begin{abstract}
The technology of light-sensitive amorphous chalcogenide layers, made from As-Ge-Se raw glasses, thick layers of acrylic polymers, made from mixture of isomers as well as two types of nanocomposites of these materials with gold nanoparticles was developed. These layers were used for direct, one-step surface geometrical and optical (amplitude-phase) relief recording by laser-or electron-beams. Investigations were directed to the establishment of giant geometrical relief formation processes, which resulted elements like bumps, single lines and diffractive gratings applicable as elements of integrated optics. The mechanism of the recording processes, which include excitation of electron-hole processes, structural transformation or polymerization, as well as mass-transport component was accepted for explanation of comparable effects at light-and electron beam recording. Selection of the materials and recording conditions with best resulting parameters was done.
\end{abstract}

\section{INTRODUCTION}

Development of active and passive elements of photonics, which can be applied also in nanoelectronics, plasmonics stimulate intensive investigations of new materials, structures as well as new technology and fabrication methods of these elements. A rather wide number of amorphous (glassy and polymer) materials are in the focus of increasing interest nowadays because of versatility, broad possibilities of targeted variations of their parameters and simpler, cheaper manufacturing in comparison with crystalline ones. Their applications in optoelectronics are mostly connected with large-scale changes of optical characteristics, their modulation by a number of external influences (electrical, optical, thermal fields) parallel with possible changes of dimensions, structure of the given element (lens, diffractive element, waveguide). Therefore, in the last decade the development of light-sensitive chalcogenide glasses and polymers for photonic applications was directed not only to the creation of new compositions but also to the goal-oriented fabrication of layered nanocomposites like amorphous chalcogenideamorphous chalcogenide, or amorphous chalcogenidemetal $[1,2]$, as well as nanocomposites with gold nanoparticles (GNP) [3,4]. Gold nanoparticles (GNP) and nanocomposite structures with GNP are of special interest because of the presence of plasmon resonance in a visible, green-red spectral range or even at longer wavelengths, depending on the form and dimensions of the GNPs. We had shown that GNPs influence the structural transformations not only in chalcogenide glasses, called sometimes as "inorganic polymers", but also in acrylate-based organic materials $[3,4]$. Therefore in the present paper we present data on optical recording, based on formation of surface patterns holographic gratings in samples, based on these materials. These investigations were made in order to develop more efficient materials and recording processes, based on the deeper insight to the mechanism of structural transformation process, including stimulated mass-transport. As fare they are connected with electron processes at least at the initial stage of excitation, we started parallel investigations and compared results with e-beam recording, since both methods are well known and used in lithography-like technology of prototype elements for photonic applications. Both mentioned methods are interesting as data source of basic research (diffusion, interdiffusion at nano-scale, the role of electron-hole transitions, influence of localized plasmon fields on structural transformations in these materials). Applied results are connected with increase of the materials sensitivity, efficiency and resolution of recording, and should be 
used for improvement of the parameters of above mentioned optical elements.

\section{Methods}

Thin Se, $\mathrm{As}_{\mathrm{x}} \mathrm{Se}_{100-\mathrm{x}}(\mathrm{x}=1,6,10,20,30,40$ and 50), as well as $\mathrm{Ge}_{\mathrm{x}} \mathrm{Se}_{100-\mathrm{x}}(\mathrm{x}=20,30$ and 40) films were deposited by thermal evaporation of glass compositions in vacuum onto silica glass substrates. In chalcogenide nanocomposites the gold nanoparticles were pre-formed by annealing thin gold layer previously deposited on a substrate by sputtering. These nanoparticles with radius between $30-90 \mathrm{~nm}$ were covered by $100-800 \mathrm{~nm}$ thick layers of selected glass composition and nanocomposite layers were obtained this way. The sample composition was controlled by energy dispersive x-ray spectroscopy (EDS) measurements via Scanning Electron Microscope (SEM - Hitachi S-4300 CFE). The thickness of layers, the surface roughness and topology of the recorded reliefs were measured by Ambios XP-1 profilemeter and by atomic force microscope (AFM) (Veeco diCaliber).

Mechanically well mixed diurethane dimethacrylate ( mixture of isomers (436909 Aldrich, UDMA), Dodecanethiol functionalized gold nanoparticles (size $5 \mathrm{~nm}$ ), silica nanoparticles (size $14 \mathrm{~nm}$ ), as well 2,2-Dimethoxy-2-phenylacetophenone iniciator for $325 \mathrm{~nm}$ or Bis(cyclopentadienyl)bis[2,6-difluoro- 3(1-pyrryl)phenyl] titanium equail to IRGACURE 784 (CAS № 125051-32-3) for $532 \mathrm{~nm}$ were used for the fabrication of polymer nanocomposites with different concentration of GNP. Pure polymer samples, without GNP were prepared from the same components. The monomers were placed between transparent glass substrate and top cover layer, which rules out the influence of oxygen. 50-100 $\mu \mathrm{m}$ thick layers were fabricated this way and used for optical recording.

Optical recording was made by polarized laser beams (He-Ne laser with $\lambda=633 \mathrm{~nm}$ for recording in As-Se chalcogenides or DPSS laser with $\lambda=532 \mathrm{~nm}$ for recording in Ge-Se chalcogenides and polymer nanocomposites with GNP). Holographic gratings with different periods $(1-7 \mu \mathrm{m})$ were recorded by a common scheme of lenseless Fourier holography. 325 laser radiations were used for recording in polymer nanocomposites for comparison. Electron beam recording was performed in the SEM with focused ebeams $(15-30 \mathrm{kV}$ accelerating voltages and 3-5 $\mathrm{nA}$ currents).

\section{RESUltS AND DISCUSSION}

The efficiency of the recording, of the surface geometrical pattern formation was estimated from the data on surface relief profile heights vs. exposure (input energy), as well from the in situ optical measurements of the diffraction efficiency of the recorded holograms.

First, we checked the efficiency of holographic recording in few compositions of chalcogenide glasses and polymers. Among the best for surface relief formation were compositions, close to the pure a-Se in As-Se system with 3-25 at \% of Se. But the $\mathrm{As}_{20} \mathrm{Se}_{80}$ composition has rather high softening temperature $\mathrm{T}_{\mathrm{g}}$ near $100^{\circ} \mathrm{C}$ and is stable against crystallization effects, so it was established as one of the most applicable for laser and e-beam recording. This material was also combined with GNP in a layered system, and the increase of relief profile height at the same exposure was established (see Fig.1,b). The same experiments were done for the polymers (see Fig.1,a).
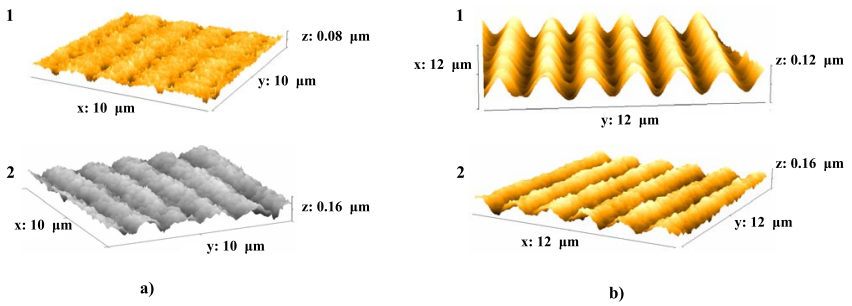

Fig. 1. AFM pictures of surface holograms a) pure polymer layer (1) and polymer nanocomposite with GNP (2), b) the same for simple $\mathrm{As}_{20} \mathrm{Se}_{80}$ layer (1) and nanocomposite with GNP (2).

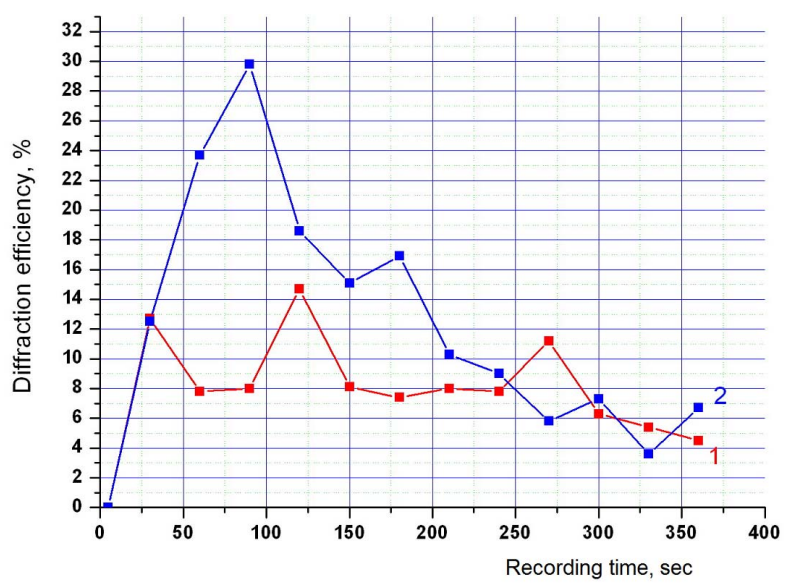

Fig. 2. Diffraction efficiency of gratings on polymer nanocomposite layers without (1) and with (2) GNP. Recording at $532 \mathrm{~nm}$, power density $9.7 \mathrm{~mW} / \mathrm{sm}^{2}$, period $1.1 \mu \mathrm{m}$ 
Essential increase of the resulting surface modulation depth and of the amplitude-phase optical modulation, diffraction efficiency of gratings were observed in polymer nanocomposites with GNP for recording with $532 \mathrm{~nm}$ irradiation, where the plasmon excitation exists and influence the structure formation (see Fig. 2 and Table 1).

Tab. 1. Diffraction efficiency (DE) and the surface modulation depth of grating $(\Delta \mathrm{H})$.

\begin{tabular}{|c|c|c|}
\hline Compositions & $\mathrm{DE}, \%$ & $\Delta \mathrm{H}, \mathrm{nm}$ \\
\hline $\mathrm{SiO}_{2}$ - monomer & 63 & 28 \\
\hline $\mathrm{GNP}_{-} \mathrm{SiO}_{2}$ - monomer & 70 & 62 \\
\hline
\end{tabular}

Thus, GNPs influence the processes of polymerization and structure formation in the nanocomposite, affecting the segregation of components and mass transport during holographic exposure. These results were used to improve the fabrication of microstructured optical elements, like photonic crystals by holographic multi beam recording (see example in Fig. 3.)

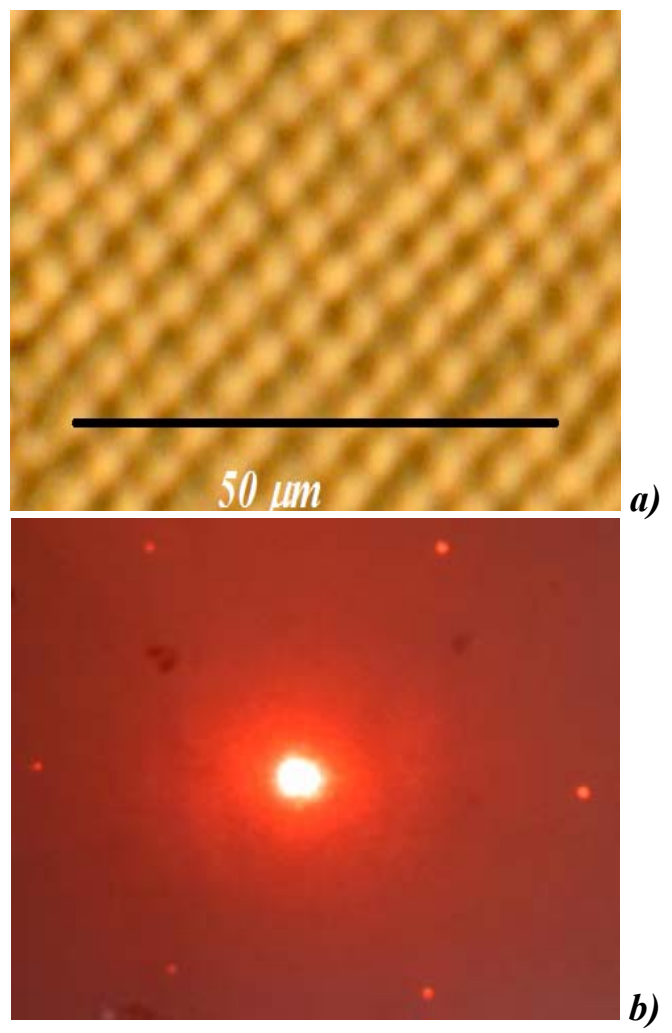

Fig. 3. The image of the photonic crystal (a) and the diffraction pattern (b).
As far the laser beam can stimulate direct surface relief formation and related optical modulation in $\mathrm{As}_{20} \mathrm{Se}_{80}$ composition after certain exposure, i.e. energy input which can excite electron-hole processes (absorption at the optical band edge of these semiconductor material), it was interesting to investigate the possibility of recording with electrons, which have much higher energy but can excite similar electron-hole processes as well, at comparable total exposures. $\mathrm{As}_{20} \mathrm{Se}_{80}$ layers with approximately 2 micron thickness on ITO covered glass substrate (to avoid charging effects) were used to investigate surface relief recording by focused e-beam. Some other compositions were tested too for comparison. Multiple line scans by e-beam focused to the spot of $50-70 \mathrm{~nm}$ diameter were performed on the sample surfaces for increasing periods of time, i.e. with increasing total exposure as presented in Fig. 4,a.
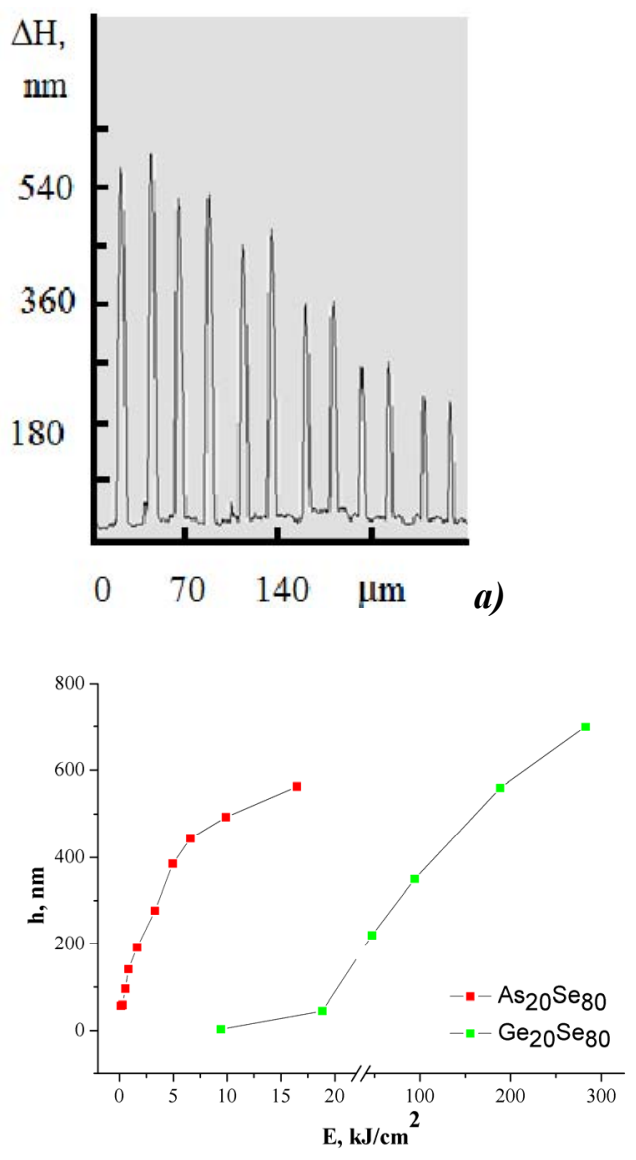

b)

Fig. 4. Height profiles of double lines recorded in $\mathrm{As}_{20} \mathrm{Se}_{80}$ layer by e-beam with decreasing exposure time (from 600 to $60 \mathrm{~s}$ ) (a). Dependence of the profile heights of e-beam recorded lines on $\mathrm{As}_{20} \mathrm{Se}_{80}$ and $\mathrm{Ge}_{20} \mathrm{Se}_{80}$ films on exposure (total submitted energy) by electrons (b). 
It was established that the $\mathrm{As}_{20} \mathrm{Se}_{80}$, as well a number of nearest glass compositions from the $\mathrm{As}_{\mathrm{x}} \mathrm{Se}_{100-\mathrm{x}}$ system with $5 \leq \mathrm{x} \leq 20$ are the best for such type of recording, as well as for the laser recording. At the same time it should be noted, that Ge-based composition, with higher softening temperature and more rigid structure are more stable but less sensitive. The comparatively large width of line sin comparison with the diameter of the recording e-beam is most probably connected with scattering profile of electrons in these materials. This effect can be reduced by decreasing the time of irradiation and the penetration depth of electrons.

Essential is the approximately same exposure (energy input) at these different kinds of excitation and relief recording, what supports the common mechanism of the recording processes in chalcogenide layers and serve the basis for development of efficient material for direct recording, prototyping photonic elements for visible and IR-spectral range. The same experiments in polymer nanocomposites, where lighelectron- and proton beam recording will be compared, are in progress.

\section{Conclusions}

Light and electron beam could be used for direct, one step process of creation of geometrical and optical patterns on thin layers, nanocomposite structures made of As-Ge-Se amorphous chalcogenide semiconductor.

The plasmon field of gold nanostructures could enhance the recording process in chalcogenide materials and in polymer nanocomposites as well.
The efficiencies of the recording, surface patterning are comparable in the investigated amorphous layers and polymer nanocomposites, but the chalcogenide layer structures are more stable, simple in handling and compatible with existing technology of microelectronics.

The created surface structures could be used in optoelectronic and photonic devices, in optical elements.

\section{ACKNOWLEDGEMENTS}

This work was supported by the 4.2.2.A$11 / 1 /$ KONV-2012-0036 project, which is co-financed by the European Union and European Social Fund and by the 2013-2020 Program aimed at maximizing the ITMO University's competitive advantage among the world's leading education centers 5/100.

\section{REFERENCES}

[1] Malyovanik, M., Ivan,S., Csik, A., Langer, G. A. Beke, D. L., Kokenyesi S., "Laser-induced optical changes in amorphous multilayers", J. Appl. Phys., 93, 2003, pp. 139-142.

[2] Takats,V., Miller, F., Jain H., Cserhati, Cs., Kokenyesi, S.,"Direct surface patterning of homogeneous and nanostructured chalcogenide layers", Phys. Status Solidi C 6, No. S1, 2009, pp. S83-S85.

[3] Charnovych, S., Szabó, I.A., Tóth A.L., Volk, J., Trunov, M.L., Kökényesi, S. "Plasmon assisted photoinduced surface changes in amorphous chalcogenide layer" Journal of Non-Crystalline Solids, vol. 377, 2013, pp. 200-204.

[4] Burunkova, J., Csarnovics, I., Denisyuk, I., Daróczi, L., Kökényesi, S. ," Enhancement of laser recording in gold/amorphous chalcogenide and gold/acrylate nanocomposite layers", Journal of Non-Crystalline Solids, vol.402, pp. 200-203 (2014). 